\title{
The auditory redundant signals effect: An influence of number of stimuli or number of percepts?
}

\author{
Hannes SChröter, Luisa S. Frei, and Rolf UlRich \\ University of Tübingen, Tübingen, Germany \\ AND \\ JEFF MiLleR \\ University of Otago, Dunedin, New Zealand
}

\begin{abstract}
In two experiments, we examined simple reaction times (RTs) for detection of the onsets and offsets of auditory stimuli. Both experiments assessed the redundant signals effect (RSE), which is traditionally defined as the reduction in RT associated with the presentation of two redundant stimuli, rather than a single stimulus. In Experiment 1, with two identical tones presented via headphones to the left ear, right ear, or both, no RSE was found in responding to tone onsets, but a large RSE was found in responding to their offsets. In Experiment 2, with a pure tone and white noise as the two stimulus alternatives, RSEs were found for responding to both onsets and offsets. The results support the notion that the occurrence of an RSE depends on the number of percepts, rather than the number of stimuli, and on the requirement to respond to stimulus onsets versus offsets. The parallel grains model (Miller \& Ulrich, 2003) provides one possible account of this pattern of results.
\end{abstract}

When participants are asked to respond as quickly as possible to the onset of any stimulus in a simple reaction time (RT) task, RT usually decreases with an increasing number of stimuli (e.g., Hershenson, 1962). This gain in RT with redundant stimuli has been termed the redundant signals effect (RSE) and has been observed with redundant stimuli within the visual (e.g., Corballis, 2002; Fischer \& Miller, 2008; Miller, 1982; Mordkoff \& Yantis, 1991, 1993; Schwarz, 1994) and the auditory (Schröter, Ulrich, $\&$ Miller, 2007) modalities, with redundant bimodal stimuli such as a tone and a light (e.g., Diederich \& Colonius, 1987; Giray \& Ulrich, 1993) or a light and an electrical pulse (e.g., Forster, Cavina-Pratesi, Aglioti, \& Berlucchi, 2002; Gondan, Lange, Rösler, \& Röder, 2004), and with redundant trimodal stimuli such as a tone, a light, and a tactile vibration stimulus (Diederich \& Colonius, 2004).

Raab (1962) explained this effect in terms of statistical facilitation. According to his race model, each stimulus is processed separately. In trials with redundant stimuli, the stimuli are processed in parallel, and a response is triggered as soon as the first stimulus is detected. Therefore, the RT is determined by the latency of a single detection process in trials with one stimulus, whereas it is determined by the winner of the parallel ongoing detection processes in trials with redundant stimuli. Since the average time of the winner in a race is usually shorter than the average detection time of each single process, this race model predicts an RT advantage for trials with redundant stimuli, as compared with trials with only one stimulus.

Subsequent research provided strong evidence for the notion that the RSE is often too large to be explained by mere statistical facilitation. Therefore, it has been suggested that the activations emerging from the redundant stimuli are somehow combined and that this combined activation triggers the response (Miller, 1982). Several quantitative models have been developed to describe this combination of information and the facilitation in RT that results from such coactivation processes (e.g., Colonius \& Arndt, 2001; Colonius \& Diederich, 2004; Miller \& Ulrich, 2003; Schwarz, 1989, 1994; Townsend \& Nozawa, 1997).

Interestingly, there is evidence that the occurrence of an RSE does not depend on the number of physical stimuli and, thus, on the number of activated receptors but, rather, on the number of percepts associated with those stimuli. In a series of simple RT experiments by Schröter et al. (2007), participants were asked to respond to the onsets of auditory stimuli that were presented via headphones to the left ear, the right ear, or both ears. In one experiment, pure tones of identical frequencies were presented to the two ears (i.e., diotic presentation). Previous research has shown that two simultaneously presented identical auditory stimuli, each delivered to one ear via headphones, are not perceived as two separate stimuli but, rather, produce the phenomenal impression of a single auditory percept 
localized between the two ears (e.g., Leakey, Sayers, \& Cherry, 1958; Odenthal, 1961, 1963; Ward, 1970). In another experiment of Schröter et al., a pure tone was presented to one ear and white noise to the other ear (dichotic presentation), which produced two separate percepts. Most important, an RSE was obtained only in the latter experiment with perceptually distinguishable stimuli, but not in the former experiment with identical stimuli, even though redundant stimuli in each of the two experiments activated receptors in both ears. Thus, these results strongly suggest that the occurrence of an RSE depends on the number of percepts, rather than on the number of stimuli. These findings also imply that the redundant target task might be a useful tool in studying processes of object perception such as binding processes. Specifically, the redundant target task could be used as an indicator of whether or not physical events are perceived as coming from a single distal object. ${ }^{1}$

\section{Overview of the Present Article}

In this article, we will first outline how the dependence of the RSE on the number of percepts, as observed by Schröter et al. (2007), can be addressed within the parallel grains model (PGM) of Miller and Ulrich (2003). ${ }^{2}$ Then we will derive predictions of an extended version of the PGM for experiments contrasting RTs to the onsets versus offsets of stimuli activating different numbers of percepts, which was designed to provide a further test of whether the RSE depends on the number of physical stimuli or the number of percepts. In brief, the predictions are that the RSE should be larger for offsets than for onsets when the stimuli activate a single percept but the RSE should be approximately the same size for onsets and offsets when the stimuli activate different percepts. Finally, we tested these predictions in two experiments. Experiment 1 employed diotically presented identical pure tones. In one block of trials, participants were asked to respond to the onsets of these tones, whereas in another block, they responded to the offsets of the same stimuli. We expected to observe virtually no RSE for responses to the onsets of these identical stimuli, which would be expected to activate a single percept, replicating the results of Schröter et al. In contrast, with responses to stimulus offsets, we expected to observe a large RSE with the same stimuli. Experiment 2 was analogous to Experiment 1 but used dichotically presented dissimilar stimuli (a pure tone and white noise). Here, we expected about the same size of RSE whether participants responded to their onsets or their offsets, because the two stimuli would activate different percepts.

\section{The Parallel Grains Model and Its Predictions}

According to the PGM (Miller \& Ulrich, 2003), the presentation of a stimulus activates a large number of functionally distinct grains, known as the grain pool. These grains may be conceptualized as different feature detectors (e.g., Burbeck \& Luce, 1982; Estes, 1950), different information codes (e.g., Treisman, 1988), or different nodes within a neural network (e.g., Anderson, 1977; McClelland, 1979). Following stimulus onset, each grain becomes activated with a certain probability, and its activation is then transmitted after a random delay to a central decision center. As soon as a criterion number, $c$, of grains have arrived at this center, the response is initiated. On redundant trials, each stimulus activates the grains in its own pool, and all the activated grains from the two stimuli are transmitted to the decision center.

The PGM suggests that the onsets of perceptually indistinguishable stimuli activate the same pool of grains. That is, when two stimuli activate the same percept, their grain pools will largely overlap. Consequently, such stimuli are predicted to produce only a small, if any, RSE, because presenting the two redundant stimuli will activate almost the same grains as activating a single one of them. In contrast, the grain pools of distinguishable stimuli that produce different percepts are expected to be disjoint or may only slightly overlap. Consequently, presenting such stimuli redundantly will activate nearly twice as many grains as presenting only one of them, leading to a large RSE. As is illustrated in the left two panels of Figure 1, the PGM therefore predicts a larger RSE with dissimilar stimuli than with similar ones when observers must respond to stimulus onset.

The left panels of Figure 1 illustrate the PGM's prediction of a larger RSE for onsets of different-percept stimuli than for those of same-percept stimuli with specific parameter values similar to those obtained in previous fits of the PGM to observed data sets. ${ }^{3}$ In order to illustrate the predictions of this simplified model, assume that each single stimulus activates $G_{1}=50$ grains. Hence, when different-percept stimuli are presented to the left and right ears on redundant trials, a total of $G_{2 \mathrm{~d}}=100$ grains is activated. By contrast, when same-percept stimuli are presented, a total of, say, $G_{2 \mathrm{~s}}=55$ grains will be activated; we assume that the grain pools do not overlap completely because the stimuli are presented to different ears. To simplify matters, the arrival time of a single grain at the decision center was modeled according to a normal distribution ( $\mu=150 \mathrm{msec}, \sigma=30 \mathrm{msec})$. From this assumption, the expected total number of active grains can be computed as a function of time, where $t=0$ defines the onset of the stimulus; a formal derivation of this function is presented in the Appendix. A response to a stimulus onset is initiated when the critical number of $c=4$ grains have reached the decision center, a criterion value that is a reasonable choice given the parameter estimates for the PGM reported by Miller and Ulrich (2003). Figure 1 shows the expected number of active grains following onsets of same-percept (upper left panel) and differentpercept (lower left panel) stimuli in single and redundant trials. Consistent with the exposition above, it can be seen that a substantial RSE is predicted for different-percept stimuli, but not for same-percept ones. In other words, the response criterion is reached more rapidly in redundant trials than in single trials only in the condition with different-percept stimuli.

The conception of stimuli as activating possibly overlapping grain pools leads to quite different predictions when observers must respond to stimulus offsets, as is illustrated in the two panels on the right of Figure 1. Al- 
Same-Percept Stimuli-Onset

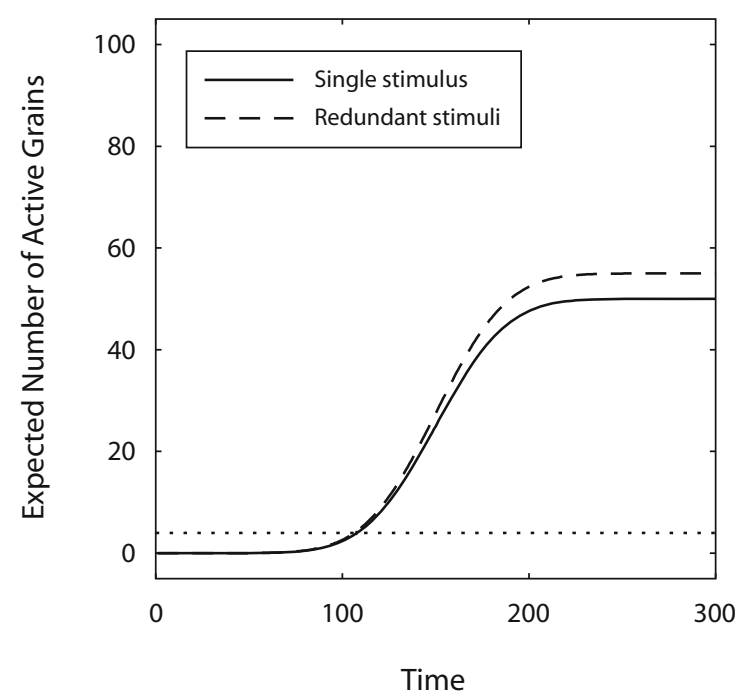

Different-Percept Stimuli-Onset

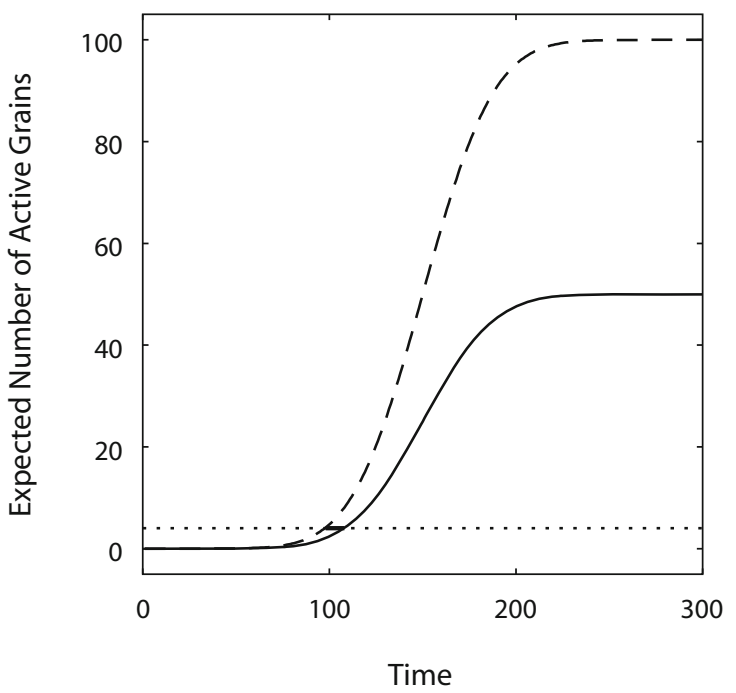

Same-Percept Stimuli-Offset

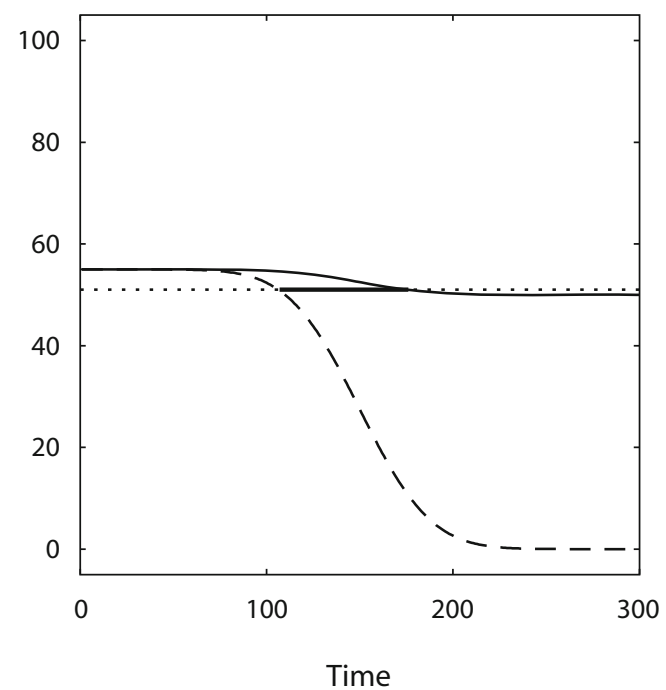

Different-Percept Stimuli-Offset

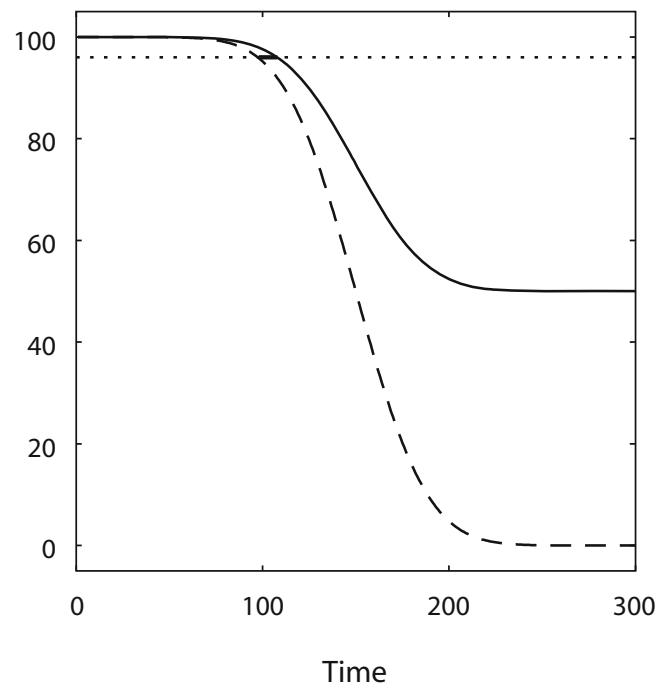

Figure 1. Predicted mean number of activated grains at time $t$ as a function of the number of stimuli (single vs. redundant), whether they are presented as onsets or offsets, and whether they activate a single percept (same-percept stimuli) or distinguishable percepts (different-percept stimuli). A response is triggered as soon as the response criterion indicated by the dotted line is crossed. In the onset conditions, this happens when the fourth grain is activated at the central decision center. In the offset conditions, it happens when the fourth grain is deactivated. As is discussed in the text, a much larger redundant signals effect (RSE) is predicted in the offset condition than in the onset condition for same-percept stimuli, but similar RSEs are predicted for these conditions with different-percept stimuli.

though the original version of the PGM did not explicitly consider predictions for responses to stimulus offsets, the model's simple framework can easily be extended to this case by incorporating the additional assumption that the response to a stimulus offset is triggered when a critical number of grains previously activated by stimulus presentation become deactivated following stimulus offset. That is, in this task, the response is assumed to be initiated when the number of grains decreases across the response criterion (indicated by the dotted line in Figure 1), rather than when it increases across the response criterion, as happens with onset stimuli.

More specifically, this extension to the PGM incorporates the idea that all grains are active during the initial stimulus presentation. Using the same stimulus parameters as those assumed for the onset stimuli in Figure 1, for example, 55 grains are active before stimulus offset when two same-percept stimuli are presented, whereas 100 grains are active before stimulus offset when two differentpercept stimuli are presented to the ears. Following stimu- 
lus offset, some or all of these grains will be deactivated. Specifically, with same-percept stimuli, $G_{1 \mathrm{~s}}=5$ grains will be deactivated following a single stimulus offset, and $G_{2 \mathrm{~s}}=55$ grains will be deactivated following a redundant stimulus offset. With different-percept stimuli, $G_{1 \mathrm{~d}}=50$ grains and $G_{2 \mathrm{~d}}=100$ grains will be deactivated following single and redundant offsets, respectively. For simplicity, we assume that the deactivation time of a single grain follows the same distribution as the activation time and that a response is triggered as soon as the number of activated grains decreases by the criterion number of $c=4$. The right panels of Figure 1 show the expected number of active grains following offsets of same-percept (upper right panel) and different-percept (lower right panel) stimuli on single and redundant trials. Consistent with the exposition above, it can be seen that a substantial RSE is predicted with offsets for both same-percept and different-percept stimuli.

In conclusion, the analysis above shows how the PGM can explain the previous finding of larger RSEs when observers respond to the onsets of different-percept stimuli than when they respond to those of same-percept stimuli (Schröter et al., 2007). This analysis further shows that a direct extension of the PGM will also predict that a very different pattern of results should be observed when observers respond to the offsets of such stimuli. Specifically, the model predicts an interaction of the following form: The onset and offset detection tasks should produce quite different RSEs with same-percept stimuli, yet they should produce rather similar RSEs with different-percept stimuli. The following two experiments were designed to evaluate this prediction.

\section{EXPERIMENT 1}

In Experiment 1, a simple RT task was used, and participants had to respond to tone onsets in one half of the experiment and to tone offsets in the other half. In order to eliminate interstimulus contingencies (see Mordkoff \& Yantis, 1991) that could influence performance on redundant trials, the probability of a tone onset (offset) in each ear was $p=.6$, independently of whether there was a tone onset (offset) in the other ear. Accordingly, responses were required on $84 \%$ of all trials; the remaining $16 \%$ were catch trials.

\section{Method}

Participants. In Experiment 1, 40 students (29 of them female) from the University of Tübingen participated in a single 45-min session as partial fulfillment of course requirements or in return for $€ 7$. Their average age was 26.0 years (range, $18-55$ years), and all but 2 claimed to be right-handed. Two additional students participated, but their data were excluded from analysis due to false alarm rates above $10 \%$.

Apparatus and Stimuli. A pure tone with a frequency of $500 \mathrm{~Hz}$ and an intensity level of $65 \mathrm{~dB}$ SPL, presented via headphones (Sony MDR-CD570), served as a stimulus. As was outlined in the introduction, these tones fuse into a single percept when presented simultaneously to the left and right ears (diotical presentation). To avoid abrupt stimulus onsets (offsets), stimulus intensity was increased (decreased) exponentially, resulting in a rise (decay) time of $50 \mathrm{msec}$ when a tone was presented (terminated). To avoid clicks produced by the activation of the sound card, the tones were low-pass filtered by an external Butterworth filter with a cutoff frequency $(-3 \mathrm{~dB})$ of $1.25 \mathrm{kHz}$. A white plus sign $\left(0.5^{\circ} \times 0.5^{\circ}\right.$ of visual angle $)$ centrally presented on a blue background of a standard computer screen (viewing distance of $60 \mathrm{~cm}$ ) served as a fixation and warning signal. Responses were registered with a response key that was centrally located in front of the participants. The participants responded in half of the blocks with their left index finger and in the other half of the blocks with their right index finger, to control for potential interactions of side of stimulation and side of response on trials with a single stimulus (cf. the Procedure section).

Procedure. In blocks in which onset presentation was used, a trial started with the presentation of the fixation cross, which was presented throughout the trial. On go trials, $600 \mathrm{msec}$ after the onset of the fixation cross, a tone was presented to the left ear, to the right ear, or to both ears. The participants were asked to press the response key as soon as they detected the onset of any tone. On catch trials, no tone was presented, and the participants were to refrain from responding. The presentation of the fixation cross (and the presentation of tones on go trials) was terminated by a response or after a maximal duration of 2,134 msec. Following the offset of the fixation cross, feedback was provided as follows: "Correct" was presented for 1,500 msec after a hit or a correct rejection; "respond only to the onset of a tone" was presented for 3,500 msec after a false alarm or a hit with an RT below 80 msec (anticipation); and "too late" was presented for $1,500 \mathrm{msec}$ when no response was registered within $1,000 \mathrm{msec}$ after tone onset (miss). The next trial started after an intertrial interval of $900 \mathrm{msec}$. After every 50 trials, the participants received accuracy feedback.

Each experimental block in which onset presentation was used comprised 100 randomly ordered trials. The participants were required to respond on 84 trials (go trials). A tone was presented exclusively to the left or to the right ear on 24 go trials each. On 36 go trials, a tone was presented simultaneously to both ears. On 16 trials, no tone was presented (catch trials), and the participants were asked to withhold the response on these trials. Each participant first performed a practice block with 17 trials and then performed an experimental block, using either the left or the right hand to respond. Then each participant performed another practice block with 17 trials and an experimental block, using the other hand to respond.

The design and procedure in blocks in which offset presentation was used were identical to those in blocks in which onset presentation was used, except for the following differences. At the beginning of each trial in blocks in which offset presentation was used, the tone was presented to both ears simultaneously with the presentation of the fixation cross. On go trials, $600 \mathrm{msec}$ after the onset of the fixation cross, the tone was terminated on the left ear, the right ear, or both ears. The participants were asked to press the response key as soon as they detected the offset of any tone. On catch trials, no tone was terminated, and the participants were asked to refrain from responding. The presentation of the fixation cross (and the presentation of any remaining tone) was terminated by a response or after a maximal duration of $2,134 \mathrm{msec}$.

Each participant performed both the onset and the offset conditions and, thus, was tested on a total of 400 trials. The order of stimulus presentation (onset-offset vs. offset-onset) and the order of response hands (left-right vs. right-left) were counterbalanced across participants.

\section{Results and Discussion}

On the basis of a preliminary inspection of the RT distribution, trials with RTs shorter than 120 msec or longer than $750 \mathrm{msec}$ were considered outliers and, thus, excluded from the data analysis. The outlier rate was low for both fast responses (1.5\%, including anticipations) and slow responses $(1.2 \%$, including misses). However, both 
Table 1

Mean Reaction Times (in Milliseconds) for Responses to Tone Onsets and Tone Offsets in Experiment 1

\begin{tabular}{cccccc}
\hline & \multicolumn{5}{c}{ Stimulus } \\
\cline { 2 - 6 } Presentation & Single & Single & Average & Faster \\
Onset & 286 & 276 & 281 & 274 & 274 \\
Offset & 300 & 281 & 291 & 275 & 256 \\
\hline
\end{tabular}

Note-Average single: Average across trials with a single stimulus presented to either the left ear or the right ear.

the rate of fast responses $[2.8 \%$ vs. $0.2 \% ; t(39)=5.20$, $p<.01]$ and the rate of slow responses $[1.3 \%$ vs. $0.2 \%$; $t(39)=3.30, p<.01]$ were higher in the offset than in the onset task. The false alarm rate on catch trials was also low $(1.4 \%)$, with a higher rate in the offset task $(2.0 \%)$ than in the onset task $(0.8 \%)[t(39)=3.13, p<.01]$.

Redundant signals effects were assessed by comparing the faster of the two single-stimulus conditions with the redundant conditions, because an $\mathrm{RT}$ advantage for the redundant conditions over the average of the two singlestimuli conditions could be an artifact of averaging across some participants who detected the left stimulus earlier and other participants who detected the right stimulus earlier (see Biederman \& Checkosky, 1970). Table 1 summarizes the mean RTs for responses to tone onsets and tone offsets in Experiment 1.

An ANOVA with the within-subjects factors of redundancy (faster single vs. redundant) and stimulus presentation (onset vs. offset) was performed on RT. The faster single stimulus was assessed separately for tone onsets and offsets for each participant. This ANOVA revealed a significant effect of redundancy $\left[F(1,39)=8.58, M S_{\mathrm{e}}=\right.$ $384.01, p<.01]$. The $\mathrm{RT}$ for redundant stimuli (265 msec) was $9 \mathrm{msec}$ shorter than that to the faster single stimu- lus $(274 \mathrm{msec})$. The effect of stimulus presentation was not significant $\left[F(1,39)=1.26, M S_{\mathrm{e}}=2,146, p>.05\right]$, suggesting that there was no systematic RT difference between responses to tone onsets $(274 \mathrm{msec})$ and responses to tone offsets $(265 \mathrm{msec})$. Most important, the interaction of redundancy and stimulus presentation was significant $\left[F(1,39)=8.62, M S_{\mathrm{e}}=391.49, p<.01\right]$. Paired $t$ tests revealed that the direction of this interaction was in line with the predictions of the PGM. Specifically, there was no significant RSE for responses to tone onsets $(0 \mathrm{msec})$ $[t(39)=0.08, p>.05]$, replicating the previous results of Schröter et al. (2007). However, there was a considerable RSE of $19 \mathrm{msec}$ for responses to tone offsets [ $t(39)=$ $3.01, p<.01]$. Because we did not observe an RSE in the onset condition, it is clear that binaural loudness summation (Marks, 1978) is not sufficient to produce an RSE (see Schröter et al., 2007).

To assess potential redundancy effects at the distributional level, Vincentized RT distributions were computed for the single- and redundant-stimulus conditions (Ulrich, Miller, \& Schröter, 2007) separately for tone onsets and offsets. Figure 2 shows the cumulative density functions (CDFs) for redundant stimuli and the sum of the Vincentized single-stimulus CDFs used to test the race model inequality.

To be consistent with the race model inequality, the redundant-stimulus CDFs would have to be everywhere below and to the right of the sum of the single-stimulus CDFs. As one might anticipate from the mean RT results, paired $t$ tests conducted at each of the 20 percentile points revealed that the present data for tone onsets did not violate this inequality. However, there were significant violations of the inequality for tone offsets. RT to redundant stimuli was reliably shorter than RT for the sum of the single CDF curves at the 2.5th $(p<.01)$ and 7.5 th $(p<$

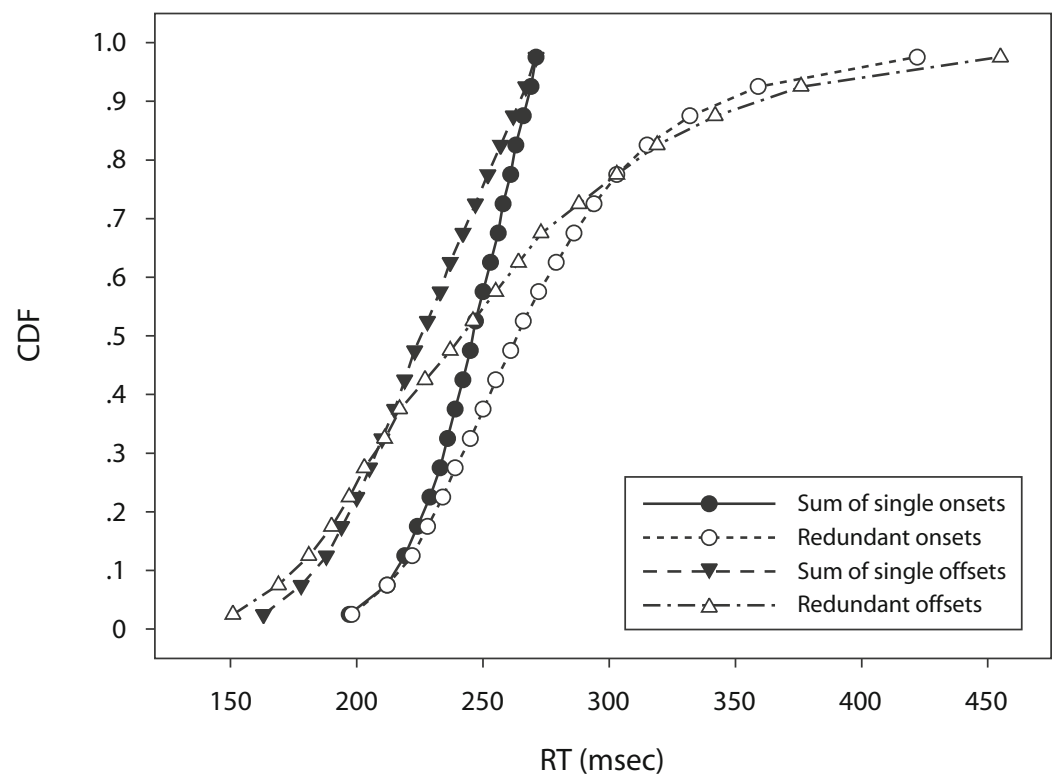

Figure 2. Estimated cumulative density functions (CDFs) of reaction times (RTs) as a function of redundancy and stimulus presentation in Experiment 1. 
$.05)$ percentiles, with $p=.056$ at the 12.5 th percentile. Therefore, there were small but significant violations of the race model inequality at low percentiles for responses to tone offsets.

From Figure 2, it is also evident that within-subjects RT variability is larger with responses to offsets than with those to onsets. This was true for the faster single-stimulus trials (average within-subjects SDs of RT equal to 79.5 vs. $48.7 \mathrm{msec}$ in the offset and onset conditions, respectively), as well as for redundant-stimulus trials (79.9 vs. $55.6 \mathrm{msec}$ ) shown in the figure. It is not plausible that this differential variability was entirely responsible for the differential redundancy gain for responses to onsets versus offsets, however, for two reasons. First, although increased variability in single-stimulus trials might enhance the RSE effect (i.e., on mean RT) by increased statistical facilitation for offset than for onset responses, there was absolutely no evidence of any RSE for the onset task despite the presence of substantial RT variability on single onset trials. If the RSE in the offset condition were entirely due to statistical facilitation, it should have been present in the onset condition, because there was plenty of variability to support it in the single-stimulus RT distribution for this condition. Second and perhaps more critically, variability per se does not influence violations of the race model inequality, because this inequality represents an upper bound on the amount of statistical facilitation. Since inequality violations were observed for the offset task, but not for the onset task, it is difficult to attribute the difference in obtained RSEs entirely to differences in RT variability.

In summary, the results of Experiment 1 clearly support the prediction of different RSEs for responses to the onsets versus offsets of two physically distinct stimuli that are perceived as one event, as is illustrated in the top two panels of Figure 1. Crucially, an RSE and evidence of coactivation were observed with such stimuli for responses to offsets, but not for responses to onsets.

\section{EXPERIMENT 2}

This experiment was identical to Experiment 1, except that the pure tone of $500 \mathrm{~Hz}$ was always presented to one ear and white noise was always presented to the other ear. The pure tone and white noise stimuli were used so that the two stimuli in this experiment could not be fused into a single percept when presented simultaneously, one to each ear (see Schröter et al., 2007).

\section{Method}

Forty students from the University of Tübingen served as participants ( 32 of them female). Their average age was 24.4 years (range, $18-51$ years), and all but 4 claimed to be right-handed. Six additional students participated, but their data were excluded from analysis due to false alarm rates above $10 \%$. The apparatus and stimuli used were identical to those in Experiment 1, except that a $500-\mathrm{Hz}$ tone was always presented to one ear and white noise ${ }^{4}$ was always presented to the other ear. For half of the participants, the tone was presented to the left ear, and the white noise was presented to the right ear. For the other half of the participants, the stimulus-to-ear mapping was reversed. Again, the participants were instructed to respond to the onset (offset) of any auditory stimulus and to withhold their re-
Table 2

Mean Reaction Times (in Milliseconds) for Responses to Stimulus Onsets and Offsets in Experiment 2

\begin{tabular}{cccccc}
\hline & \multicolumn{5}{c}{ Stimulus } \\
\cline { 2 - 6 } Presentation & $\begin{array}{c}\text { Single } \\
\text { Left }\end{array}$ & $\begin{array}{c}\text { Single } \\
\text { Right }\end{array}$ & $\begin{array}{c}\text { Average } \\
\text { Single }\end{array}$ & $\begin{array}{c}\text { Faster } \\
\text { Single }\end{array}$ & Redundant \\
\hline Onset & 316 & 317 & 316 & 299 & 287 \\
Offset & 297 & 285 & 291 & 276 & 225 \\
\hline
\end{tabular}

Note-Average single: Average across trials with a single stimulus presented to either the left ear or the right ear.

sponse when no stimulus was presented (terminated) in blocks in which onset (offset) stimuli were used.

\section{Results and Discussion}

As in Experiment 1, trials with RTs shorter than $120 \mathrm{msec}(2.3 \%$, including anticipations) or longer than $800 \mathrm{msec}(0.9 \%$, including misses) were considered outliers and, thus, excluded from the data analysis. Again, both the rate of fast responses $[4.1 \%$ vs. $0.5 \% ; t(39)=5.46$, $p<.01]$ and the rate of slow responses $[0.3 \%$ vs. $0.2 \%$; $t(39)=2.15, p<.05]$ were higher in the offset than in the onset task. The false alarm rate on catch trials $(2.4 \%)$ was low, with a higher rate in the offset task (4.5\%) than in the onset task $(0.3 \%)[t(39)=7.64, p<.01]$. Table 2 summarizes the mean RTs for responses to auditory stimulus onsets and offsets in Experiment 2.5

An ANOVA with the within-subjects factors of redundancy (faster single vs. redundant) and stimulus presentation (onset vs. offset) was performed on RT. As in Experiment 1, the ANOVA revealed a significant effect of redundancy $\left[F(1,39)=80.69, M S_{\mathrm{e}}=480.72, p<\right.$ $.001]$. Mean RT was $31 \mathrm{msec}$ less for redundant stimuli $(256 \mathrm{msec})$ than for the faster single stimulus $(287 \mathrm{msec})$. In contrast to Experiment 1, the effect of stimulus presentation was significant $\left[F(1,39)=34.48, M S_{\mathrm{e}}=2,112\right.$, $p<.001]$, reflecting shorter responses to stimulus offsets $(250 \mathrm{msec})$ than to onsets $(293 \mathrm{msec})$. Again, the interaction of redundancy and stimulus presentation was significant $\left[F(1,39)=37.89, M S_{\mathrm{e}}=412.08, p<.001\right]$. Contrary to the predictions of the PGM, the RSE was larger for responses to stimulus offsets $(51 \mathrm{msec})$ than for those to onsets $(12 \mathrm{msec})$. In line with the predictions of the PGM, however, paired $t$ tests revealed that significant RSEs were observed for both offsets $[t(39)=8.00, p<$ $.001]$ and onsets $[t(39)=5.57, p<.001]$.

To assess potential redundancy effects at the distributional level, we again computed Vincentized RT distributions for the single- and redundant-stimulus conditions (see Ulrich et al., 2007) separately for stimulus onsets and offsets. Figure 3 shows the redundant-stimulus CDFs and the sum of the Vincentized single-stimulus CDFs used to test the race model inequality.

Again, $t$ tests were conducted at each of the 20 percentile points. Replicating the results of Schröter et al. (2007), there were significant violations of the inequality for stimulus onsets. The RT to redundant stimuli was reliably less $(p<.05)$ than the RT for the sum of the single CDF curves at the 7.5th and the 12.5th percentiles (both $p$ s $<.05$ ), with $p=.064$ at the 2.5 th percentile. 


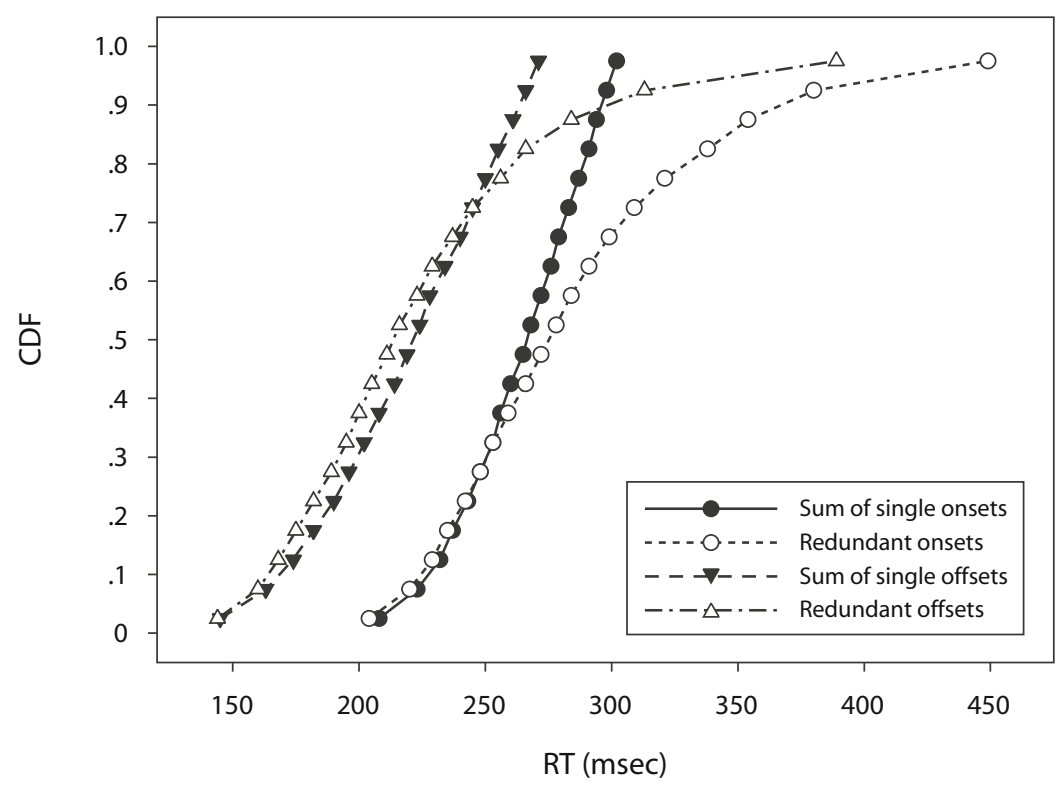

Figure 3. Estimated cumulative density functions (CDFs) of reaction times (RTs) as a function of redundancy and stimulus presentation in Experiment 2.

Therefore, there were small but significant violations of the race model inequality at low percentiles for responses to stimulus onsets. As one might anticipate from the mean RT results for stimuli offsets, the race model inequality was violated for a large range of percentiles with these stimuli. The CDF for redundant stimuli was reliably $(p<$ .05 ) above the sum of the single CDFs for the 12.5th up to the 47.5th percentiles.

In summary, as was predicted by the simplified version of the PGM, RSEs (and evidence for coactivation) were observed for responses to both onsets and offsets of dichotically presented stimuli. However, contrary to the predictions, the RSE was much larger for responses to stimulus offsets.

\section{GENERAL DISCUSSION}

The present study investigated RSEs for responses to the onsets and offsets of same-percept and different-percept auditory stimuli in order to investigate an account of the previous finding that the occurrence of an RSE depends on the number of activated percepts, rather than on the number of presented stimuli (or the number of stimulated receptors; Schröter et al., 2007). In general, the results of the two experiments replicated the previous findings with onset stimuli that an RSE is found only when the two redundant stimuli activate two separate percepts, but not when they are fused into a single percept. On the basis of the idea that this happens because same-percept stimuli activate the same grains or units within the perceptual system (see Miller \& Ulrich, 2003), it was predicted from the PGM that large RSEs would be found with same-percept stimuli, as well as with different-percept stimuli, when participants responded to stimulus offsets.
In Experiment 1, the participants were asked to respond to the onsets or offsets of identical tones presented to the left and right ears (i.e., diotically presented stimuli). These stimuli are known to fuse into a single percept when presented simultaneously (e.g., Leakey et al., 1958). As in previous work, no RSE or evidence of coactivation was observed when the participants responded to the onsets of these stimuli. In contrast, a strong RSE and evidence of coactivation were found when the participants responded to their offsets. This pattern-RSEs with responses to offsets of identical stimuli, but not with responses to their onsets - is in accordance with the predictions of the PGM.

In Experiment 2, the participants responded to onsets or offsets of dichotically presented stimuli (i.e., a pure tone and a white noise stimuli), which are too different to be fused into a single percept (see Schröter et al., 2007). In contrast to Experiment 1, RSEs and evidence of coactivation were observed for responses to both onsets and offsets.

We used the PGM (Miller \& Ulrich, 2003) as a conceptual framework to illustrate specifically how these effects might be explained. In terms of the PGM, approximately the same grains are activated whether one or two samepercept stimuli are presented because the grain pools of the two stimuli largely overlap. In the onset detection task, then, the decision center receives almost the same input whether a single stimulus or two stimuli are presented, so there is little or no extra activation on redundant trialshence, little or no RSE. In the offset detection task, however, only a few grains are deactivated when there is a single stimulus offset, because the remaining stimulus continues to activate almost all of the grains that were activated when the two stimuli were presented at the start of the trial. Essentially, all grains are deactivated when both 
stimuli are terminated, however. Thus, redundant offsets lead to a far greater change in activation than do single offsets. The RSE is quite large for offset detection with these stimuli, then, because redundant offsets produce a far faster decay of activation than does a single offset.

In contrast, within the PGM, different-percept stimuli would activate fairly disjoint pools of grains. The onset or offset of either stimulus would then activate or deactivate a large number of grains, regardless of the presence of the other stimulus. RSEs are found for responses to both onsets and offsets with different-percept stimuli, then, because redundant changes activate or deactivate about twice as many grains as do single changes in both cases.

Although the general pattern of results observed in Experiments 1 and 2 is in line with the predictions of the PGM, it must be noted that one specific result was unexpected and, thus, appears to be at variance with this account. More precisely, a much larger RSE was observed for offsets than for onsets with different-percept stimuli (Experiment 2). Here, RSEs of similar size were predicted for onsets and offsets because the patterns of activation are virtually identical in both conditions. On the basis of this version of the PGM, then, it is surprising that redundant offsets of different-percept stimuli produced such a large RSE.

This unexpected finding can be explained by a reasonable ad hoc elaboration of the PGM in which the decision criterion $c$ differed between the onset and offset detection tasks in Experiment 2. One possibility is that the ongoing stimulation before the imperative offset might have produced a high level of arousal (e.g., Niemi, 1979; Sollers \& Hackley, 1997; Ulrich \& Mattes, 1996), especially because two distinct auditory stimuli were being presented simultaneously. The possibility of increased arousal is supported by the finding of a larger false alarm rate in the offset condition than in the onset condition of this experiment. This arousal could have decreased the signal-to-noise ratio in this condition. In order to avoid premature responses due to this decreased ratio, the participants could have chosen a larger value of $c$ in the offset than in the onset task (see Näätänen, 1971; Näätänen \& Merisalo, 1977). Such a differential criterion setting could have produced the larger RSE for offsets than for onsets in Experiment 2, because the RSE tends to increase with $c$ in the PGM.

It is interesting to contrast the present results with auditory stimuli against previous examinations of RSEs to the onsets versus offsets of visual stimuli. Fischer and Miller (2008) studied simple RTs to the onsets or offsets of squares on one or both sides of fixation, and they found smaller RSEs to offsets than to onsets. Clearly, then, the present findings of larger RSEs to offsets than to onsets is specific to the auditory modality, rather than reflecting some general property of processing stimulus transients. In terms of the PGM, the finding of an RSE with identical visual stimuli on different sides of fixation is not unexpected, of course, because such stimuli would activate different percepts by virtue of being in different locations.

Like the results of Schröter et al. (2007), the present results reinforce the idea that the redundant target task can be used as a tool to tap into processes that are involved in object perception, such as binding processes. Specifically, this task can elucidate whether or not auditory events are perceived as emerging from a single or several distal sources. Specifically, the RSE appears to be found only in the latter case with responses to stimulus onsets, although in both cases with responses to offsets.

In summary, the present study employed the redundant signals paradigm to assess RSEs for responses to the onsets versus offsets of auditory stimuli. This design allowed us to investigate an account for the finding that with stimulus onsets, the occurrence of the RSE depends on the number of percepts, rather than on the number of stimuli (i.e., the number of stimulated receptors). Specifically, an account of this finding in terms of a simple version of the PGM predicts further that the RSE should depend on the number of stimuli, rather than on the number of percepts, when responses are made to stimulus offsets rather than to onsets. The results of the present experiments are in accord with this prediction.

\section{AUTHOR NOTE}

This research was supported by a grant from the Deutsche Forschungsgemeinschaft (SCHR 1180/2-1). The authors thank Toby Mordkoff and two anonymous reviewers for constructive comments on a preliminary version of the manuscript. Correspondence concerning this article may be addressed to H. Schröter, Abteilung für Allgemeine und Biologische Psychologie, Psychologisches Institut, Universität Tübingen, Friedrichstr. 21, 72072 Tübingen, Germany (e-mail: hannes.schroeter@, uni-tuebingen.de).

\section{REFERENCES}

Anderson, J. A. (1977). Neural models with cognitive implications. In D. LaBerge \& S. Samuels (Eds.), Basic processes in reading: Perception and comprehension (pp. 27-90). Hillsdale, NJ: Erlbaum.

Biederman, I., \& Checkosky, S. F. (1970). Processing redundant information. Journal of Experimental Psychology, 83, 486-490.

Burbeck, S. L., \& LucE, R. D. (1982). Evidence from auditory simple reaction times for both change and level detectors. Perception \& Psychophysics, 32, 117-133.

Colonius, H., \& ARndT, P. (2001). A two-stage model for visualauditory interaction in saccadic latencies. Perception \& Psychophysics, 63, 126-147.

Colonius, H., \& Diederich, A. (2004). Multisensory interaction in saccadic reaction time: A time-window-of-integration model. Journal of Cognitive Neuroscience, 16, 1000-1009.

Corballis, M. C. (2002). Hemispheric interactions in simple reaction time. Neuropsychologia, 40, 423-434.

DIEDERICH, A. (1992). Intersensory facilitation: Race, superposition, and diffusion models for reaction time to multiple stimuli. Frankfurt: Lang.

DIEDERICH, A. (1995). Intersensory facilitation of reaction time: Evaluation of counter and diffusion coactivation models. Journal of Mathematical Psychology, 39, 197-215.

Diederich, A., \& Colonius, H. (1987). Intersensory facilitation in the motor component? A reaction time analysis. Psychological Research, 49, 23-29.

Diederich, A., \& Colonius, H. (2004). Bimodal and trimodal multisensory enhancement: Effects of stimulus onset and intensity on reaction time. Perception \& Psychophysics, 66, 1388-1404.

Estes, W. K. (1950). Toward a statistical theory of learning. Psychological Review, 57, 94-107.

Fischer, R., \& Miller, J. O. (2008). Differential redundancy gain in onset detection versus offset detection. Perception \& Psychophysics, 70, 431-436.

Forster, B., Cavina-Pratesi, C., Aglioti, S. M., \& Berlucchi, G. (2002). Redundant target effect and intersensory facilitation from 
visual-tactile interactions in simple reaction time. Experimental Brain Research, 143, 480-487.

Giray, M., \& UlRICH, R. (1993). Motor coactivation revealed by response force in divided and focused attention. Journal of Experimental Psychology: Human Perception \& Performance, 19, 1278-1291.

Gondan, M., Lange, K., Rösler, F., \& RöDer, B. (2004). The redundant target effect is affected by modality switch costs. Psychonomic Bulletin \& Review, 11, 307-313.

Hershenson, M. (1962). Reaction time as a measure of intersensory facilitation. Journal of Experimental Psychology, 63, 289-293.

Leakey, D. M., Sayers, B. M., \& Cherry, C. (1958). Binaural fusion of low- and high-frequency sounds. Journal of the Acoustical Society of America, 30, 222.

MARKS, L. E. (1978). Binaural summation of the loudness of pure tones. Journal of the Acoustical Society of America, 64, 107-113.

MCClelland, J. L. (1979). On the time relations of mental processes: An examination of systems of processes in cascade. Psychological Review, 86, 287-330.

MiLleR, J. (1982). Divided attention: Evidence for coactivation with redundant signals. Cognitive Psychology, 14, 247-279.

MilleR, J. (1998). Cupid: A program for computations with probability distributions. Behavior Research Methods, Instruments, \& Computers, 30, 544-545.

Miller, J., \& UlRich, R. (2003). Simple reaction time and statistical facilitation: A parallel grains model. Cognitive Psychology, 46, 101-151.

MoRdKofF, J. T., \& YANTIS, S. (1991). An interactive race model of divided attention. Journal of Experimental Psychology: Human Perception \& Performance, 17, 520-538.

MoRdKoff, J. T., \& YANTIS, S. (1993). Dividing attention between color and shape: Evidence of coactivation. Perception \& Psychophysics, $\mathbf{5 3}, 357-366$.

NÄ̈̈TÄNEN, R. (1971). Non-aging fore-periods and simple reaction time. Acta Psychologica, 35, 316-327.

NÄÄTÄNEN, R., \& MERISALO, A. (1977). Expectancy and preparation in simple reaction time. In S. Dornic (Ed.), Attention and performance VI (pp. 115-138). Hillsdale, NJ: Erlbaum.

NiEMI, P. (1979). Stimulus intensity effects on auditory and visual reaction processes. Acta Psychologica, 43, 299-312.

Odenthal, D. W. (1961). Simultaneous dichotic frequency discrimination. Journal of the Acoustical Society of America, 33, 357-358.

Odenthal, D. W. (1963). Perception and neural representation of simultaneous pure tone stimuli. Acta Physiologica et Pharmacologica Neerlandica, 12, 453-496.

RAAB, D. H. (1962). Statistical facilitation of simple reaction times. Transactions of the New York Academy of Sciences, 24, 574-590.

SCHröter, H., UlRich, R., \& Miller, J. (2007). Effects of redundant auditory stimuli on reaction time. Psychonomic Bulletin \& Review, 14, 39-44.

Schwarz, W. (1989). A new model to explain the redundant-signals effect. Perception \& Psychophysics, 46, 498-500.

Schwarz, W. (1994). Diffusion, superposition, and the redundanttargets effect. Journal of Mathematical Psychology, 38, 504-520.

SOlleRs, J. J., III, \& HACKLEY, S. A. (1997). Effects of foreperiod duration on reflexive and voluntary responses to intense noise bursts. Psychophysiology, 34, 518-526.

Townsend, J. T., \& Nozawa, G. (1997). Serial exhaustive models can violate the race model inequality: Implications for architecture and capacity. Psychological Review, 104, 595-602.
Treisman, A. M. (1988). Features and objects: The fourteenth Bartlett memorial lecture. Quarterly Journal of Experimental Psychology, 40A, 201-237.

Ulrich, R., \& Mattes, S. (1996). Does immediate arousal enhance response force in simple reaction time? Quarterly Journal of Experimental Psychology, 49A, 972-990.

UlRich, R., Miller, J., \& SCHRÖTER, H. (2007). Testing the race model inequality: An algorithm and computer programs. Behavior Research Methods, 39, 291-302.

WARD, W. D. (1970). Musical perception. In J. V. Tobias (Ed.), Foundations of modern auditory theory (Vol. 1, pp. 405-447). New York: Academic Press.

\section{NOTES}

1. We thank Toby Mordkoff for suggesting this possibility.

2. It would also be possible to apply a similar analysis to other stochastic models of the RSE (e.g., Diederich, 1992, 1995; Schwarz, 1989, 1994). In contrast to these models, however, the framework of the PGM provides an especially simple conceptualization of the impact of the number of percepts on the RSE. It is for this reason that the following analysis is based on the PGM. Because the same issue might be conceptualized in other models, however, the present approach does not aim at distinguishing between these models, nor does it imply a rigorous test of the PGM's core assumptions. Hence, the purpose of the following analysis was primarily to illustrate the major conceptual issues involved in accounting for the influence of the number of percepts on the RSE.

3 . Figure 1 illustrates the time differences for detection needed in terms of the average activation functions. The average detection time needed, however, depends on more complicated order statistics, as is explained at the end of the Appendix. For the following arguments, this difference can be neglected.

4. The white noise was generated by MATLAB with a bandwidth of $0-44.1 \mathrm{kHz}$, and it had the same intensity and envelope as the pure tone. It was also filtered externally by the Butterworth filter, reducing the signal strength for frequencies above the cutoff frequency of $1.25 \mathrm{kHz}$ by $3 \mathrm{~dB}$ per octave. Therefore, the presented stimulus was a mixture of white noise (below the cutoff frequency) and pink noise (above the cutoff frequency).

5. To be consistent with Experiment 1, Table 2 shows the mean RTs for left and right single stimuli. Of course, the two single stimuli in Experiment 2 could alternatively be defined by stimulus identity (tone, white noise), rather than by side of presentation. Importantly, because the stimulus-to-ear mapping was fixed for each participant, the RT for the faster single stimulus is the same whether the two single stimuli are defined by side of presentation or by identity. Therefore, the main RT analysis does not depend on the definition of the two single stimuli. The same holds true for the distributions of the sum of single-stimuli CDFs. A separate ANOVA with within-subjects factors of stimulus identity (single tone, single noise) and stimulus presentation (onset, offset) revealed that responses to single tone stimuli $(295 \mathrm{msec})$ were shorter than responses to single noise stimuli $(312 \mathrm{msec})\left[F(1,39)=15.04, M S_{\mathrm{e}}=\right.$ $776.67, p<.001]$. Furthermore, this effect was modulated by stimulus presentation $\left[F(1,39)=11.22, M S_{\mathrm{e}}=664.32, p<.01\right]$. The RT advantage for responses to tones was larger in blocks with onset presentation $(31 \mathrm{msec})$ than in blocks with offset presentation $(4 \mathrm{msec})$.

\section{APPENDIX \\ Formal Derivation of Predicted Relationship Between Time and Expected Number of Activated Grains}

Here, we will derive the PGM's predictions concerning the expected number of active grains at time $t$ separately for conditions in which observers respond to stimulus onsets and offsets. First, we will derive the prediction for the onset condition.

Assume that a total of $G$ grains may be activated by stimulus onset. It should be noted that this derivation applies to single and redundant trials, because the value of $G$ simply differs between these two types of trials. Furthermore, let the random variable $X_{i}$ represent the arrival time of grain $i,(i=1, \ldots, G)$, at the decision center. The cumulative probability distribution (CDF) associated with $X_{i}$ is $F_{i}(t)$. For the sake of simplicity, we will assume that the latency distributions of all grains are identical - that is, $F=F_{1}=F_{2}=\ldots=F_{G}$. 


\section{APPENDIX (Continued)}

Let $I_{i}(t)$ be an indicator variable that indexes whether grain $i$ has been activated by time $t$ after stimulus onset. Specifically, let $I_{i}(t)=1$ if grain $i$ is active by time $t$ and let $I_{i}(t)=0$ if it has not been activated by time $t$. Thus, the number $N(t)$ of active grains at time $t$ is given by

$$
N(t)=\sum_{i=1}^{G} I_{i}(t)
$$

Consequently, the expected number of grains at time $t$ is computed by

$$
\begin{aligned}
E[N(t)] & =E\left[\sum_{i=1}^{G} I_{i}(t)\right] \\
& =\sum_{i=1}^{G} E\left[I_{i}(t)\right] \\
& =\sum_{i=1}^{G}\left[1 \cdot P\left(X_{i} \leq t\right)+0 \cdot P\left(X_{i}>t\right)\right] \\
& =\sum_{i=1}^{G} F_{i}(t) \\
& =G \cdot F(t) .
\end{aligned}
$$

An analogous derivation applies to the offset condition. In this case, the random latency $X_{i}$ represents the time to deactivate grain $i$ after stimulus offset. Therefore, the indicator variable indexes whether grain $i$ has been deactivated by time $t$ or not. Thus $I_{i}(t)=1$ when grain $i$ is deactivated by time $t$, and $I_{i}(t)=0$ if it is still active by time $t$. Consequently, the expected number of active grains at time $t$ is given by

$$
\begin{aligned}
E[N(t)] & =E\left[\sum_{i=1}^{G} I_{i}(t)\right] \\
& =\sum_{i=1}^{G}\left[0 \cdot P\left(X_{i} \leq t\right)+1 \cdot P\left(X_{i}>t\right)\right] \\
& =\sum_{i=1}^{G}\left[1-F_{i}(t)\right] \\
& =G \cdot[1-F(t)] .
\end{aligned}
$$

It should be noted that the mean activation functions derived here and depicted in Figure 1 do not directly reflect the mean latencies of detection times. For each condition, the mean detection time is determined by the $c$ th order statistic of the activation times across all grains - that is, by $X_{c: G}$ (see Miller \& Ulrich, 2003). Nevertheless, for the example presented in the introduction and displayed in Figure 1, the latencies illustrated by the activation functions yield virtually the same predictions concerning the RSE as the ones derived from order statistics.

Specifically, for the onset task, no grains are active before stimulus onset. In both the same-percept and different-percept conditions, the onset of a single stimulus will activate 50 grains. For these stimuli and a decision criterion of $c=4$ grains, the expected detection time is computed with CUPID (Miller, 1998) to be $E\left[X_{4: 50}\right]=106.1 \mathrm{msec}$. Redundant same-percept stimuli will activate 55 grains, resulting in a mean of $E\left[X_{4: 55}\right]=104.6 \mathrm{msec}$, whereas this mean is $E\left[X_{4: 100}\right]=96.0 \mathrm{msec}$ with different-percept redundant stimuli, for which a total of 100 grains will be activated. Therefore, the predicted RSE for same-percept onset stimuli is negligible (i.e., $106.1-104.6=1.5 \mathrm{msec}$ ), whereas a substantial RSE is predicted for different-percept onset stimuli (i.e., $106.1-96.0=10.1 \mathrm{msec}$ ).

In contrast, for the offset task, a stimulus offset is detected as soon as $c=4$ grains have been deactivated following stimulus offset. In the same-percept condition, only 5 grains from the active pool of 55 grains will be deactivated when one of the two stimuli is switched off, resulting in a theoretical mean latency of $E\left[X_{4: 5}\right]=164.9$ msec. When both stimuli are switched off, all 55 grains will be deactivated, and the corresponding theoretical mean is $E\left[X_{4: 55}\right]=104.6 \mathrm{msec}$. In the different-percept condition, the active grain pool (100 grains) is larger than that in the same-percept condition ( 55 grains). Fifty grains will be deactivated when one of the two stimuli is switched off, and all grains will again be deactivated on redundant trials. Hence, the theoretical means in the different-percept condition are $E\left[X_{4: 50}\right]=106.1 \mathrm{msec}$ and $E\left[X_{4: 100}\right]=96.0 \mathrm{msec}$ for single and redundant trials, respectively. Therefore, a very large RSE is predicted for same-percept offset stimuli (i.e., $164.9-104.6=60.3 \mathrm{msec}$ ), whereas a smaller RSE is predicted for different-percept offset stimuli (i.e., $106.1-96.0=10.1 \mathrm{msec}$ ). 